01

\title{
Применение теории возмущений к исследованию возникновения квантовых вихрей, образующихся при воздействии сверхкороткого импульса электромагнитного поля на атом
}

\author{
(C) Н.В. Ларионов, ${ }^{1}$ С.Ю. Овчинников, ${ }^{2}$ А.А. Смирновский, ${ }^{1,2}$ А.А. Шмидт ${ }^{2}$ \\ ${ }^{1}$ Санкт-Петербургский политехнический университет Петра Великого, \\ 195251 Санкт-Петербург, Россия \\ ${ }^{2}$ Физико-технический институт им. А.Ф. Иофффе РАН, \\ 194021 Санкт-Петербург, Россия \\ e-mail: smirta@mail.ru
}

(Поступило в Редакцию 4 мая 2018 г.)

Методом нестационарной теории возмущений исследованы механизмы образования квантовых вихрей, возникающих при ионизации водородоподобного атома сверхкоротким импульсом лазерного излучения. Показано, что эти вихри обусловлены эффектами квантовой интерференции.

DOI: 10.21883/JTF.2018.11.46621.177-18

\section{Введение}

Одной из нетривиальных особенностей в динамике квантовых систем является появление структур, которые могут трактоваться как вихри. В недавней работе [1] экспериментально наблюдалось возникновение вихреподобных образований при распространении лазерного излучения в конденсате Бозе-Эйнштейна. Ранее подобные явления были исследованы в ряде работ при помощи численного моделирования [2,3]. Образующиеся вихреподобные структуры, которые по форме близки к известной гидродинамической дорожке Кармана [4,5], соответствуют изолированным нулям волновой функции. Квантовые вихри могут также наблюдаться в экспериментах как локальные (запрещенные) области, в которых квантовые частицы не могут быть обнаружены. При определенных воздействиях, в силу волновой природы элементарных частиц, аналогичные вихревые структуры могут появляться и в простых одноэлектронных квантовых системах [6,7]. Изучение свойств таких вихрей и способов их генерации представляет собой интерес, как с фундаментальной, так и с практической точки зрения. В квантово-информационных приложениях, где используются источники неклассических состояний полевой [8] или атомной систем [9], перспективными могут оказаться источники состояний скоррелированных между собой вихрей. Исследования динамики вихрей электронной плотности, возникающих при воздействии сверхкоротких лазерных импульсов на квантовую систему, могут быть востребованы для диагностики быстрых процессов в веществе [10].

Анализ процесса возникновения вихрей и их эволюции удобно проводить на модельных задачах, для которых можно получить численные и аналитические решения. Одной из таких задач является хорошо известная проблема ионизации одиночного одноэлектронного атома импульсом лазерного излучения. В работе [11] нами численно исследовалась пространственно-временная эволюция квантовой системы, образованной взаимодействием сверхкороткого импульса классического электромагнитного поля (длительность от нескольких десятков до несколько сотен аттосекунд) с водородоподобным атомом в двумерном приближении. В этой работе показано, что при таком воздействии на систему возникают особые точки (изолированные нули волновой функции), которые в гидродинамической интерпретации трактуются как квантовые вихри, идентифицированные нами при анализе пространственного распределения электронной плотности и плотности потока вероятности для электрона. Путем преобразования переменных, обеспечивающего переход к эволюции в так называемом расширяющемся пространстве, проанализирована электронная плотность в сопряженном импульсном пространстве, где также присутствуют вихревые структуры, которые могут перемещаться в неизменном виде на макроскопические расстояния [12]. Это обеспечивает возможность экспериментального детектирования таких структур.

Численный расчет на основе нестационарного уравнения Шредингера включает в себя весь спектр процессов в эволюции исследуемой квантовой системы, но не всегда позволяет выявить физические механизмы рассматриваемых явлений. Поэтому особый интерес представляет отыскание приближенных аналитических решений задачи, которые могут прояснить физическую картину процесса. В работе [11] на основе борновского приближения также были получены предварительные аналитические результаты, которые показали, что для описания возникновения квантовых вихрей необходима реализация теории возмущений более высоких порядков.

\section{1. Анализ возникновения квантовых вихрей методом нестационарной теории возмущений}

\section{1. Постановка модельной задачи}

В настоящей работе мы попытаемся описать физические механизмы возникновения квантовых вихрей, осно- 

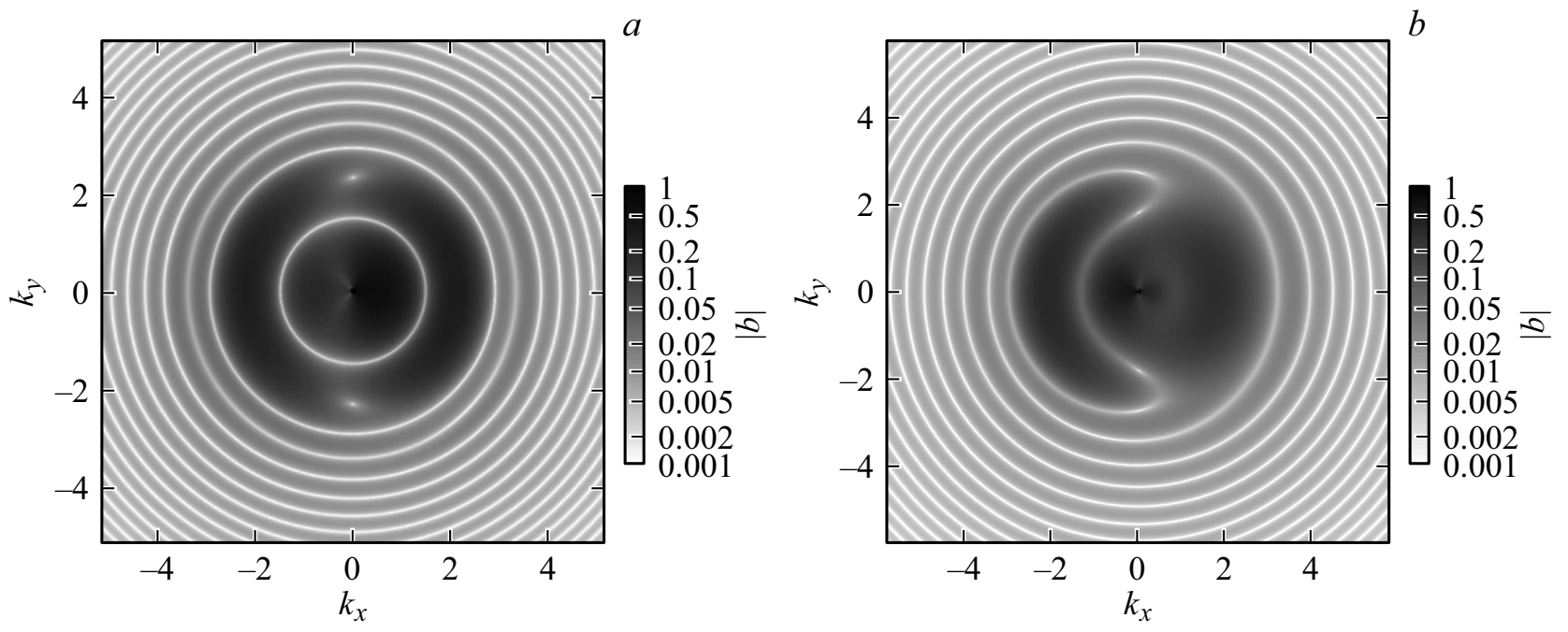

Рис. 1. Распределение по импульсам ионизированного электрона $|b|=\left|b\left(k_{x}, k_{y}\right)\right|$, построенное для больших времен $t \gg T$. Логарифмический масштаб. Параметры импульса: $\tilde{E}_{0}=0.5, \omega=\pi, T: a-4, b-3$.

вываясь на нестационарной теории возмущений $[13,14]$. Как и ранее в [11], будем рассматривать взаимодействие двумерного атома водорода $[15,16]$ с импульсом классического электромагнитного поля. Оператор Гамильтона этой системы записывается в виде суммы Гамильтониана свободного атома $\hat{H}_{0}$ и оператора взаимодействия $\hat{V}(t)$ :

$$
\begin{gathered}
\hat{H}=\hat{H}_{0}+\hat{V}(t), \\
\hat{H}_{0}=\frac{1}{2} \hat{p}^{2}+U(\mathbf{r}), \\
U(\mathbf{r})=-\frac{1}{2 \rho}, \\
\hat{V}(t)=-\hat{\mathbf{d}} \cdot \mathbf{E}=-\rho \cos (\varphi) \tilde{E}(t),
\end{gathered}
$$

где $\rho$ и $\varphi$ - полярные компоненты вектора $\mathbf{r}$, $\mathbf{E}=\mathbf{e}_{x} \tilde{E}(t)$ - напряженность электрического поля, поляризованного вдоль оси $x, \hat{\mathbf{d}}=\mathbf{r}$ - оператор дипольного момента, а в кулоновском потенциале $U(\mathbf{r})$ берется половинный заряд электрона (здесь и далее для простоты используются атомные единицы: $\left.e=\hbar=m_{e}=1\right)$. Зависимость амплитуды поля от времени дается следующим выражением:

$$
\tilde{E}(t)=\tilde{E}_{0} \cos (\omega t)[\theta(T-t)-\theta(-t)],
$$

где $\theta(t)$ - функция Хевисайда, $\omega$ - частота, $T-$ длительность импульса и $\tilde{E}_{0}-$ амплитуда колебаний. Предполагается, что длительность импульса $T$ содержит малое число периодов поля, однако приближенно можно выделить несущую частоту $\omega$, которая берется выше энергии ионизации.

Для дальнейшего сравнения с аналитическими решениями используются результаты численного моделирования, которые наглядно демонстрируют возникновение квантовых вихрей в пространстве волновых чисел ионизированного электрона. На рис. 1, для больших времен $t \gg T$ и разных параметров импульса, приведены графики модуля амплитуды вероятности $b\left(k_{x}, k_{y}\right)$, описывающей распределение импульсов ионизированного электрона. При длительности импульса $T=4$ отчетливо видно два вихря, расположенных на оси $k_{y}$ симметрично относительно начала координат. При нечетном значении $T=3$ наблюдается уже по два вихря в каждой из полуплоскостей, расположенных на оси $y$.

Предварительные аналитические оценки, основанные на первом порядке теории возмущений, не позволили обнаружить квантовые вихри. Стало очевидным, что для их идентификации необходимо использовать теорию возмущений, в которой бы учитывались переходы в конечное состояние через различные промежуточные состояния. Как известно, такие переходы могут быть учтены, начиная со второго порядка теории возмущений. Ниже рассматривается применение теории возмущений для решения поставленной модельной задачи и приводится сравнение полученных аналитических и численных результатов.

\section{2. Ионизация во втором порядке теории возмущений}

Для построения рядов теории возмущений необходимо знать явные выражения для собственных функций и собственных значений оператора $\hat{H}_{0}[15,16]$. Некоторые из этих величин, их аппроксимации и используемые в настоящей работе матричные элементы дипольного момента перехода приведены в приложении. Здесь для удобства отметим, что собственные состояния непрерывного спектра $\left|\Psi_{k m}^{(0)}\right\rangle$ характеризуются модулем волнового вектора $k$, определяющего энергию $E_{k}=$ $=k^{2} / 2=\left(k_{x}^{2}+k_{y}^{2}\right) / 2$, и проекцией момента на ось 
$z: \quad m=0, \pm 1, \pm 2, \ldots$. Соответствующие волновые функции в координатном представлении $\Psi_{k m}^{(0)}(\rho, \varphi)=$ $=R_{k|m|}(\rho) \Phi_{m}(\varphi)$ являются так называемыми кулоновскими волнами, где $R_{k|m|}(\rho), \Phi_{m}(\varphi)=e^{i m \varphi} / \sqrt{2 \pi}-$ радиальная и угловая части соответственно.

В нашей задаче рассматривается случай надбарьерной ионизации в условиях мгновенного воздействия, поэтому будем пренебрегать возможностью ионизации посредством переходов через промежуточные состояния дискретного спектра. Таким образом, из дискретного спектра нам понадобится только основное состояние $\left|\Psi_{10}^{(0)}\right\rangle$, в котором атом был приготовлен в начальный момент времени $t=0$, где нижние индексы соответствуют главному квантовому числу $n=1\left(E_{1}=-0.5\right)$ и проекции момента $m=0$. В координатном представлении волновая функция основного состояния имеет вид

$$
\Psi_{10}^{(0)}(\rho, \varphi)=R_{10}(\rho) \Phi_{0}(\varphi)=\sqrt{\frac{2}{\pi}} e^{-\rho} .
$$

Запишем уравнение Шредингера в представлении взаимодействия

$$
i \frac{\partial|\Psi(t)\rangle}{\partial t}=\hat{V}^{I}(t)|\Psi(t)\rangle,
$$

где $|\Psi(t)\rangle-$ вектор состояния. Оператор взаимодействия в представлении взаимодействия записывается в виде

$$
\hat{V}^{I}(t)=e^{i \hat{H}_{0} t} \hat{V}(t) e^{-i \hat{H}_{0} t} .
$$

Считая, что воздействие является слабым, решение уравнения (6) может быть записано в виде следующего итерационного ряда:

$$
\begin{aligned}
& |\Psi(t)\rangle=|\Psi(0)\rangle+(-i) \int_{0}^{t} \hat{V}^{I}\left(t^{\prime}\right)|\Psi(0)\rangle d t^{\prime} \\
& +(-i)^{2} \int_{0}^{t} \hat{V}^{I}\left(t^{\prime}\right) \int_{0}^{t^{\prime}} \hat{V}^{I}\left(t^{\prime \prime}\right)|\Psi(0)\rangle d t^{\prime \prime} d t^{\prime}+(-i)^{3} \\
& \times \int_{0}^{t} \hat{V}^{I}\left(t^{\prime}\right) \int_{0}^{t^{\prime}} \hat{V}^{I}\left(t^{\prime \prime}\right) \int_{0}^{t^{\prime \prime}} \hat{V}^{I}\left(t^{\prime \prime \prime}\right)|\Psi(0)\rangle d t^{\prime \prime \prime} d t^{\prime \prime} d t^{\prime}+\ldots,
\end{aligned}
$$

где в качестве нулевого приближения используется значение волновой функции в начальный момент времени $t=0,|\Psi(0)\rangle \equiv\left|\Psi_{10}^{(0)}\right\rangle$.

Спроецируем (8) на собственное состояние оператора $\hat{H}_{0}$, соответствующее непрерывному спектру $\left|\Psi_{k m}^{(0)}\right\rangle$. Тогда, после стандартного суммирования итерационного ряда, уравнение для определения амплитуды вероятности обнаружения электрона в соответствующем состоянии непрерывного спектра $b_{k m, 10}(t)=\left\langle\Psi_{k m}^{(0)} \mid \Psi(t)\right\rangle$ будет выглядеть следующим образом:

$$
\begin{aligned}
& b_{k m, 10}(t)=(-i) \int_{0}^{t} V_{k m, 10}^{I}\left(t^{\prime}\right) d t^{\prime} \\
& +(-i) \sum_{m^{\prime}} \int_{0}^{\infty} k^{\prime} d k^{\prime} \int_{0}^{t} V_{k m, k^{\prime} m^{\prime}}^{I}\left(t^{\prime}\right) b_{k^{\prime} m^{\prime}, 10}\left(t^{\prime}\right) d t^{\prime},
\end{aligned}
$$

где второй нижний индекс 10 у амплитуды указывает на состояние системы в начальный момент времени. $V_{k m, k^{\prime} m^{\prime}}^{I}(t)=V_{k m, k^{\prime} m^{\prime}}(t) \exp \left(i \omega_{k k^{\prime}} t\right)$ - матричный элемент оператора возмущения, $\omega_{k k^{\prime}}=\left(E_{k}-E_{k^{\prime}}\right)-$ частота перехода.

Физический смысл первого слагаемого в (9) - это амплитуда вероятности „однофотонного“ перехода из начального $\left|\Psi_{10}^{(0)}\right\rangle$ в конечное состояние $\left|\Psi_{k m}^{(0)}\right\rangle$. Второе слагаемое описывает многофотонную ионизацию (начиная с ,двухфотонной“) через промежуточные состояния непрерывного $\left|\Psi_{k^{\prime} m^{\prime}}^{(0)}\right\rangle$ спектра.

Запишем явный вид матричного элемента оператора возмущений (4)

$$
\begin{aligned}
& V_{\alpha m^{\prime}, \beta m}(t)=-\tilde{E}(t) \int_{0}^{\infty} \rho d \rho \\
& \times \int_{0}^{2 \pi} d \varphi \Psi_{\alpha m^{\prime}}^{(0) *}(\rho, \varphi) \rho \cos (\varphi) \Psi_{\beta m}^{(0)}(\rho, \varphi) \\
& =-\frac{\tilde{E}(t)}{2}\left(\delta_{m^{\prime}, m+1}+\delta_{m^{\prime}, m-1}\right)\left\langle\alpha,\left|m^{\prime}\right||\rho| \beta,|m|\right\rangle,
\end{aligned}
$$

где введено следующее обозначение для радиальной части матричного элемента дипольного момента перехода:

$$
\left\langle\alpha,\left|m^{\prime}\right||\rho| \beta,|m|\right\rangle=\int_{0}^{\infty} \rho d \rho R_{\alpha\left|m^{\prime}\right|}^{*}(\rho) \rho R_{\beta|m|}(\rho),
$$

и индексы $\alpha, \beta$ могут относится как к дискретному $n=1$, так и к непрерывному $k$-спектрам. Здесь при интегрировании по углу $\varphi$ было использовано свойство ортогональности угловых функций $\Phi_{m}(\varphi)$ :

$$
\int_{0}^{2 \pi} \Phi_{m}^{*}(\varphi) \Phi_{m^{\prime}}(\varphi) d \varphi=\delta_{m m^{\prime}}
$$


Используя (10), уравнение для амплитуды (9) можно переписать в следующем виде:

$$
\begin{aligned}
b_{k m, 10}(t)= & \frac{i}{2}\left(\delta_{m, 1}+\delta_{m,-1}\right)\langle k, 1|\rho| 1,0\rangle \int_{0}^{t} \tilde{E}\left(t^{\prime}\right) e^{i \omega_{k 1} t^{\prime}} d t^{\prime} \\
& +\frac{i}{2} \sum_{m^{\prime}=m \pm 1} \int_{0}^{\infty} k^{\prime} d k^{\prime}\left\langle k,|m||\rho| k^{\prime},\left|m^{\prime}\right|\right\rangle \\
& \times \int_{0}^{t} \tilde{E}\left(t^{\prime}\right) e^{i \omega_{k k^{\prime}} t^{\prime}} b_{k^{\prime} m^{\prime}, 10}\left(t^{\prime}\right) d t^{\prime}
\end{aligned}
$$

Далее будем использовать приближение свободного электрона, что соответствует замене радиальной части кулоновской волны $R_{k|m|}(\rho)$, представленной вырожденной гипергеометрической функцией, на функцию Бесселя первого рода $\widetilde{R}_{k,|m|}(\rho)=$ $=J_{|m|}(k \rho)$ (радиальная часть цилиндрической волны $\left.\widetilde{\Psi}_{k m}^{(0)}(\rho, \varphi)=\widetilde{R}_{k|m|}(\rho) \Phi_{m}(\varphi)\right)$. Условие нормировки для $\widetilde{R}_{k,|m|}(\rho)=J_{|m|}(k \rho)[17]$ остается точно таким же, как и для точной функции, и имеет вид

$$
\int_{0}^{\infty} \rho d \rho \widetilde{R}_{k, m}(\rho) \widetilde{R}_{k^{\prime}, m}(\rho)=\frac{1}{k} \delta\left(k-k^{\prime}\right),
$$

где $\delta(x)$ - дельта-функция Дирака.

Это приближение позволяет легко вычислить матричные элементы (см. приложение), входящие в уравнение (12), и провести в нем интегрирование по всем промежуточным состояниям с волновыми числами $k^{\prime}$. После этих преобразований уравнение (12) упростится и будет иметь следующий вид

$$
\begin{aligned}
b_{k m, 10}(t) & =\frac{i}{2}\left(\delta_{m, 1}+\delta_{m,-1}\right) \frac{6 k}{\left(1+k^{2}\right)^{5 / 2}} \\
& \times \int_{0}^{t} \tilde{E}\left(t^{\prime}\right) e^{i \omega_{k 1} t^{\prime}} d t^{\prime}+\frac{i}{2}\left(\frac{|m|}{k^{2}} \mp \frac{1}{k} \frac{\partial}{\partial k}\right) k \\
& \times \int_{0}^{t} \tilde{E}\left(t^{\prime}\right) b_{k m-1,10}\left(t^{\prime}\right) d t^{\prime}+\frac{i}{2}\left(\frac{|m|}{k^{2}} \pm \frac{1}{k} \frac{\partial}{\partial k}\right) k \\
& \times \int_{0}^{t} \tilde{E}\left(t^{\prime}\right) b_{k m+1,10}\left(t^{\prime}\right) d t^{\prime},
\end{aligned}
$$

где верхний знак для слагаемого с $b_{k m-1,10}(t)$ соответствует значениям $m>0$, а нижний $-m \leq 0$. Для слагаемого с $b_{k m+1,10}(t)$ верхний знак используется для значений $m \geq 0$, а нижний - для $m<0$.

Нас будут интересовать амплитуды вероятности того, что ионизированный электрон после окончания действия импульса имеет определенное значение проекции импульса на ось $x$ при фиксированном нулевом значении проекции импульса на ось $y-b\left(k_{x}, k_{y}=0\right)$, и наоборот, $b\left(k_{x}=0, k_{y}\right)$. Эти амплитуды определяются через $b_{k m, 10}(T)$ следующим образом:

$$
\begin{aligned}
b\left(k_{x}, k_{y}=0\right) & =\sum_{m} \int_{0}^{\infty} k d k b_{k m, 10}(T)\left\langle k_{x} \mid \tilde{\Psi}_{k m}^{(0)}\right\rangle \\
& =\sum_{m}(i)^{-m} C_{m} b_{k_{x} m, 10}(T), \\
b\left(k_{x}=0, k_{y}\right) & =\sum_{m} \int_{0}^{\infty} k d k b_{k m, 10}(T)\left\langle k_{y} \mid \tilde{\Psi}_{k m}^{(0)}\right\rangle \\
& =\sum_{m} C_{m} b_{k_{y} m, 10}(T),
\end{aligned}
$$

где векторы $\left|k_{x}\right\rangle,\left|k_{y}\right\rangle$ соответствуют состояниям частицы с определенным значением импульса, в координатном представлении они имеют вид

$$
\phi_{k_{x}}(x)=\frac{e^{i k_{x} x}}{\sqrt{2 \pi}}, \quad \phi_{k_{y}}(y)=\frac{e^{i k_{y} y}}{\sqrt{2 \pi}} .
$$

При получении второго равенства в (15) использованы выражения для цилиндрических волн $\left|\tilde{\Psi}_{k m}^{(0)}\right\rangle$ в импульсном представлении, см. формулы (П3), (П4) в Приложении. При отрицательных значениях проекций волнового вектора $k_{x, y}$ в (15) заменяется на $\left|k_{x, y}\right|$. Коэффициент $C_{m}=1$ при $k_{x, y}>0, m \geq 0$ или при $k_{x, y}<0, m \leq 0$ и $C_{m}=(-1)^{m}$ при $k_{x, y}>0, m<0$ или при $k_{x, y}<0, m>0$.

Полученные уравнения (14) позволяют легко записать амплитуды (15) с точностью до нужного порядка теории возмущений. Так, с точностью до интересующего нас второго порядка амплитуды (15) запишутся в следующем виде:

$$
\begin{aligned}
b\left(k_{x}, k_{y}=0\right)= & -2 i \operatorname{sign}\left(k_{x}\right) b_{\left|k_{x}\right| 1,10}^{(1)}(T) \\
& +b_{\left|k_{x}\right| 0,10}^{(2)}(T)-2 b_{\left|k_{x}\right| 2,10}^{(2)}(T), \\
b\left(k_{x}=0, k_{y}\right)= & b_{\left|k_{y}\right| 0,10}^{(2)}(T)+2 b_{\left|k_{y}\right| 2,10}^{(2)}(T),
\end{aligned}
$$

где

$$
\begin{aligned}
b_{k m, 10}^{(1)}(t)= & \frac{i}{2}\left(\delta_{m, 1}+\delta_{m,-1}\right) \frac{6 k}{\left(1+k^{2}\right)^{5 / 2}} \int_{0}^{t} \tilde{E}\left(t^{\prime}\right) e^{i \omega_{k 1} t^{\prime}} d t^{\prime} \\
b_{k m, 10}^{(2)}(t)= & \frac{i}{2}\left(\frac{|m|}{k^{2}} \mp \frac{1}{k} \frac{\partial}{\partial k}\right) k \int_{0}^{t} \tilde{E}\left(t^{\prime}\right) b_{k m-1,10}^{(1)}\left(t^{\prime}\right) d t^{\prime} \\
& +\frac{i}{2}\left(\frac{|m|}{k^{2}} \pm \frac{1}{k} \frac{\partial}{\partial k}\right) k \int_{0}^{t} \tilde{E}\left(t^{\prime}\right) b_{k m+1,10}^{(1)}\left(t^{\prime}\right) d t^{\prime}
\end{aligned}
$$

и учтено, что $b_{k m, 10}^{(1,2)}(t)=b_{k-m, 10}^{(1,2)}(t)$. 
Сделаем несколько важных наблюдений. Из (16) видно, что амплитуда $b\left(k_{x}=0, k_{y}\right)$, которая и позволит в рассматриваемом случае идентифицировать квантовые вихри, появляется только начиная со второго порядка теории возмущений. Она содержит три амплитуды, одна из которых $b_{\left|k_{y}\right| 0,10}^{(2)}(t)$ соответствует состоянию с нулевым орбитальным моментом $m=0$ (круговая волна), а другие $b_{\left|k_{y}\right| m= \pm 2,10}^{(2)}(t)-$ состояниям с $m= \pm 2$.

Также из (15) легко видеть, что $b\left(k_{x}=0, k_{y}\right)$ будет определяться только $b_{\left|k_{y}\right| m, 10}^{(s)}(t)$ с четными $s$, т.е. следующий ненулевой вклад даст четвертый порядок теории возмущений.

\section{2. Сравнение численного и аналитического решений}

На рис. 2, для тех же параметров импульса, что и на рис. 1, $a$ построены графики модуля амплитуды $b\left(k_{x}, k_{y}=0\right)$ (см. рис. 1,a). Сплошная линия соответствует численному решению уравнения Шредингера, пунктирные - аналитическим результатам (16), полученным либо в первом, либо во втором порядке теории возмущений. Отметим некоторые наблюдаемые особенности. В области малых волновых чисел $\left(k_{x} \approx 0\right)$ расчетный результат и аналитическое решение сильно расходятся. Это связано с несколькими факторами: большая ,погрешность“ расчета в этой области, которая вызвана спецификой вышеупомянутого перехода в масштабированное пространство, а в случае аналитического рассмотрения - сделанные приближения, в частности замена кулоновской волны на цилиндрическую и пренебрежение переходами через дискретный спектр. Тем не менее в области резонансных значений $k_{x}= \pm \sqrt{2\left(\omega+E_{1}\right)} \approx \pm 2.3$ совпадение расчетных

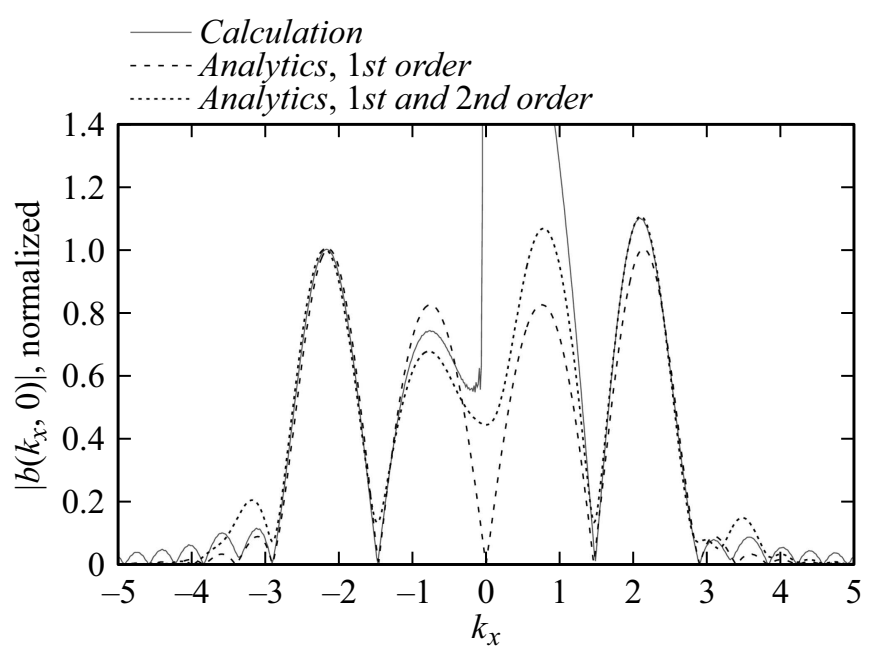

Рис. 2. Распределение $\left|b\left(k_{x}, 0\right)\right|$ при $T=4$, сравнение численного расчета (сплошная линия) с аналитическим, полученным в 1-ом порядке (штриховая линия) и в первых двух порядках (пунктирная линия) теории возмущений.

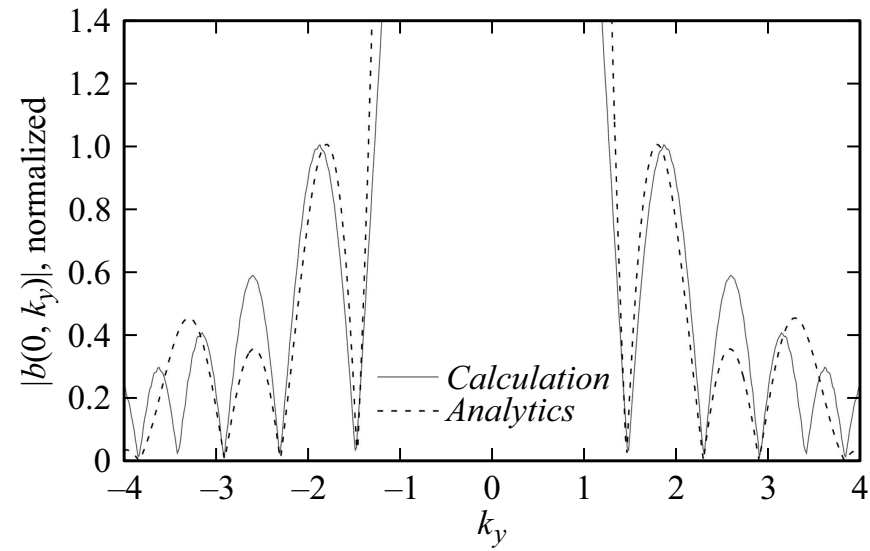

Рис. 3. Распределение $\left|b\left(0, k_{y}\right)\right|$ при $T=4$, сравнение численного расчета (сплошная линия) с аналитическим (штриховая линия).

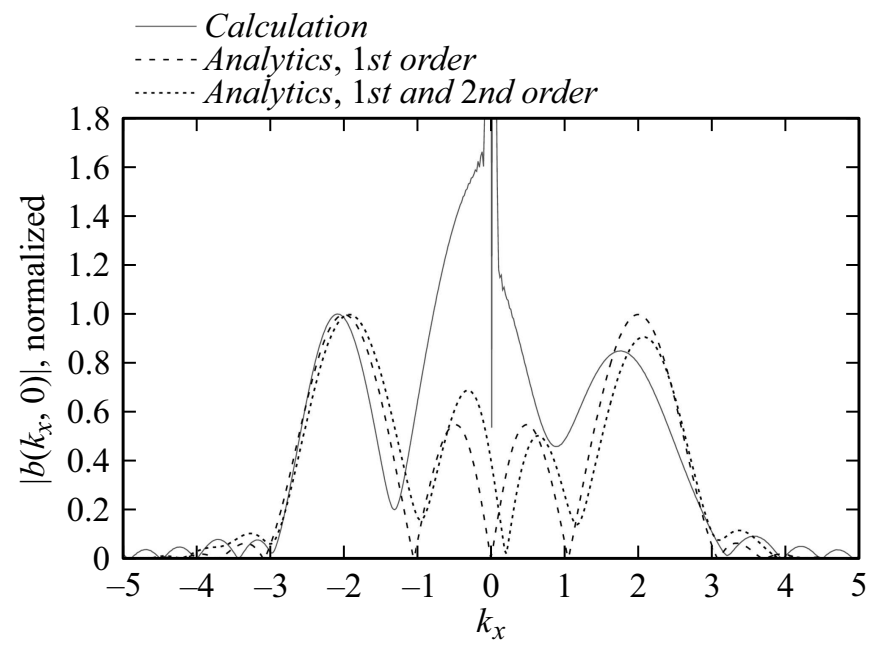

Рис. 4. Распределение $\left|b\left(k_{x}, 0\right)\right|$ при $T=3$.

результатов с аналитическими довольно хорошее, особенно при учете вкладов 2-го порядка теории возмущений. Результаты, полученные в первом порядке, не дают асимметрию в распределении относительно $k_{x}=0$, обусловленную инертностью изначально связанного электрона. Появление дополнительных пиков в аналитическом расчете при учете вкладов 2-го порядка связано с проявлением резонанса на удвоенных частотах.

Теперь рассмотрим амплитуду $\left|b\left(k_{x}=0, k_{y}\right)\right|$ (рис. 3$)$. Сравнение этого рисунка с рис. 2 показывает, что там, где у $\left|b\left(k_{x}, k_{y}=0\right)\right|$ наблюдается максимум, т.е. для $k_{x} \approx \pm 2.3$, для $\left|b\left(k_{x}=0, k_{y}\right)\right|$ имеется минимум, который, как следует из анализа рис. $1, a$, связан с наличием в этой области квантового вихря.

Для $T=3$ графики для амплитуд $\left|b\left(k_{x}, k_{y}=0\right)\right|$ и $\left|b\left(k_{x}=0, k_{y}\right)\right|$ представлены на рис. 4 и 5 соответственно. Для этого случая согласие численного моделирования и аналитического решения несколько хуже. Однако 


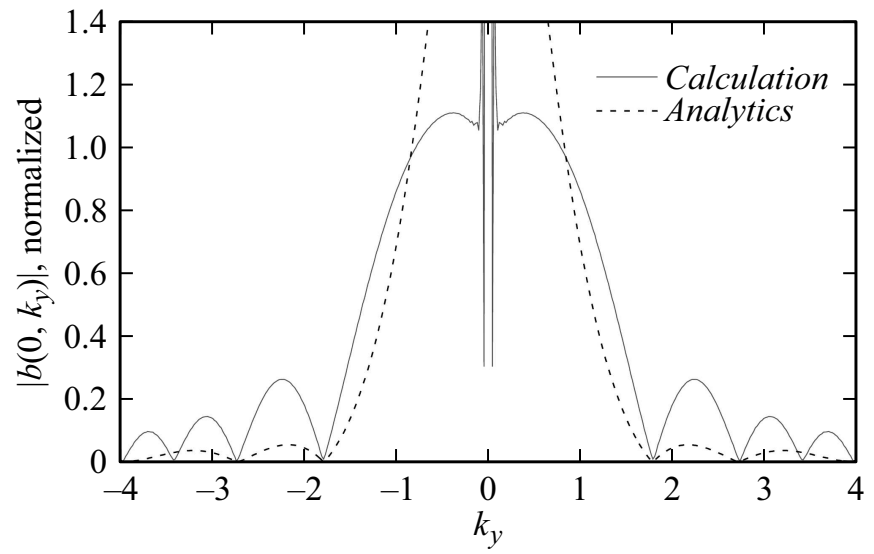

Рис. 5. Распределение $\left|b\left(k_{y}, 0\right)\right|$ при $T=3$.

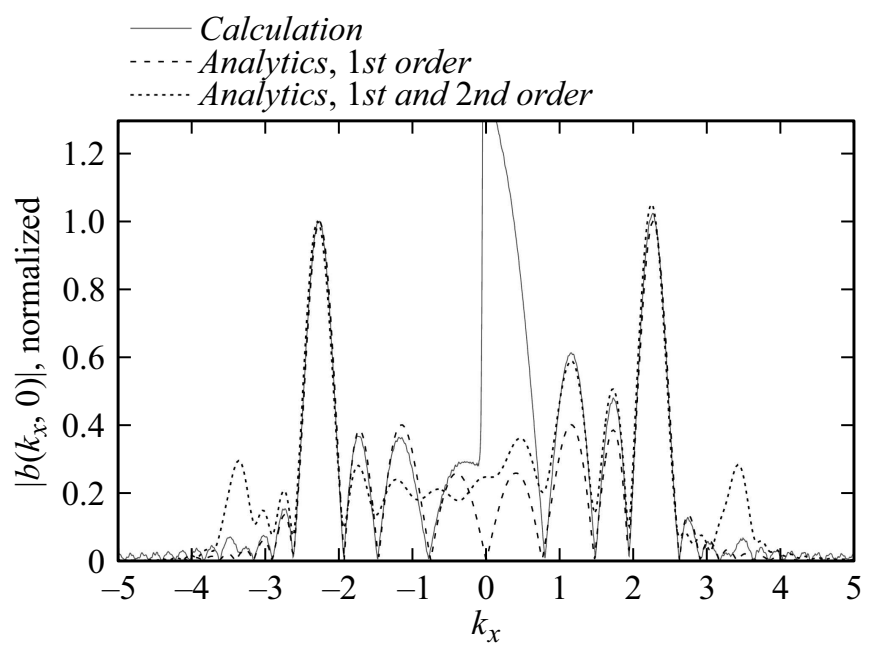

Рис. 6. Распределение $\left|b\left(k_{x}, 0\right)\right|$ при $T=8$.

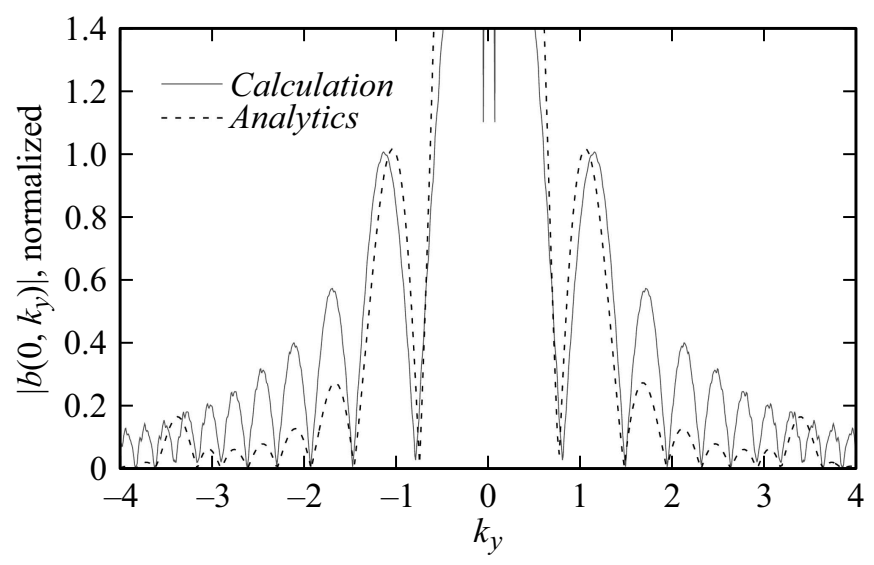

Рис. 7. Распределение $\left|b\left(0, k_{y}\right)\right|$ при $T=8$.

также, как и в предыдущем случае, четко видны области локализации квантовых вихрей. В этом случае их уже четыре (рис. $1, b$ ).
Расчеты для еще меньших $T$ показывают более сильное расхождение между результатами численного моделирования и результатами, полученными аналитически. Это расхождение связано с тем, что для столь коротких импульсов [18] использованные в работе приближения становятся некорректными. Тем не менее отметим, что, как показано в [11], в численном расчете при $T=1$ квантовых вихрей не наблюдается, что обусловлено недостаточным временем взаимодействия атома с полем.

При увеличении длительности импульса $T$ согласие аналитики и расчета в первом порядке теории возмущений улучшается, однако второй порядок дает некоторые расхождения. В качестве иллюстрации на рис. 6 и 7 приводятся амплитуды $\left|b\left(k_{x}, k_{y}=0\right)\right|$ и $\left|b\left(k_{x}=0, k_{y}\right)\right|$ при $T=8$. Так же как и в расчете, аналитика предсказывает появление квантовых вихрей в области $k_{x}=0$, $k_{y} \approx \pm 2.3$.

Таким образом, из полученных результатов можно заключить, что второй порядок теории возмущений позволяет идентифицировать квантовые вихри. Полученные аналитические выражения (16) для амплитуд вероятностей, описывающих распределение импульсов ионизированного электрона, позволяют понять природу этих вихрей. Так выражение для $b\left(k_{x}=0, k_{y}\right)$ (16) представлено тремя амплитудами, каждая из которых соответствует состоянию ионизированного электрона с определенным квантовым числом $m$. С другой стороны, каждое из этих состояний появляется в результате переходов через континуум промежуточных состояний с различными значениям момента. Интерференция этих переходов, а также фазовая корреляция между образованными в результате ионизации состояниями обеспечивает появление нулей в распределении импульсов, интерпретирующихся как центры квантовых вихрей.

\section{Заключение}

В настоящей работе методами нестационарной теории возмущений была рассмотрена задача об ионизации водородоподобного двумерного атома сверхкоротким импульсом классического электромагнитного поля, несущая частота которого соответствовала энергии, превышающей энергию ионизации.

Интерес представляли идентификация и выявление механизмов образования квантовых вихрей, ранее обнаруженных и проанализированных нами с помощью численного моделирования в работе [11]. С точностью до второго порядка теории возмущений включительно были получены аналитические выражения для амплитуд вероятностей (16), описывающих распределение импульсов ионизированного электрона; проведено сравнение с численными расчетами. Показано, что образование вихрей обусловлено эффектами квантовой интерференции. 


\section{Приложение}

Цилиндрические волны в импульсном представлении. Используя известные представления функций Бесселя [17]

$$
\begin{gathered}
J_{m}(x)=\frac{(i)^{m}}{2 \pi} \int_{0}^{2 \pi} \exp (-i x \cos (\varphi)+i m \varphi) d \varphi, \\
J_{m}(x)=\frac{1}{2 \pi} \int_{0}^{2 \pi} \exp (-i x \sin (\varphi)+i m \varphi) d \varphi,
\end{gathered}
$$

следующие свойства

$$
\begin{aligned}
& J_{-m}(x)=(-1)^{m} J_{m}(x), \\
& J_{m}(-x)=(-1)^{m} J_{m}(x),
\end{aligned}
$$

а также условие (13), можно записать цилиндрические волны в импульсном представлении

$$
\begin{gathered}
\tilde{\Psi}_{k m}^{(0)}\left(k_{x}\right)=(i)^{-m} C_{m} \frac{\delta\left(k-k_{x}\right)}{k}, \\
\tilde{\Psi}_{k m}^{(0)}\left(k_{y}\right)=C_{m} \frac{\delta\left(k-k_{y}\right)}{k},
\end{gathered}
$$

где при отрицательных значениях проекций $k_{x, y}$ заменяется на $\left|k_{x, y}\right|$. Коэффициент $C_{m}=1$ при $k_{x, y}>0, m \geq 0$ или при $k_{x, y}<0, m \leq 0$ и $C_{m}=(-1)^{m}$ при $k_{x, y}>0, m<0$ или при $k_{x, y}<0, m>0$.

Радиальная часть матричного элемента оператора возмущения $\left\langle\alpha,\left|m^{\prime}\right||\rho| \beta,|m|\right\rangle$ (10), вычисленная в приближении свободного электрона. Для перехода из основного состояния атома водорода в состояние непрерывного спектра имеем

$$
\langle k, 1|\rho| 1,0\rangle=\frac{6 k}{\left(1+k^{2}\right)^{5 / 2}} .
$$

При рассмотрении переходов в конечное состояние непрерывного спектра через промежуточные состояния того же непрерывного спектра необходимо вычислить следующие матричные элементы: $\left\langle k,|m||\rho| k^{\prime},|m \mp 1|\right\rangle$. Это можно сделать с помощью известных соотношений, выполняемых для цилиндрических функций [17]

$$
\begin{aligned}
& J_{\alpha-1}(x)=\left(\frac{\alpha}{x}+\frac{d}{d x}\right) J_{\alpha}(x), \\
& J_{\alpha+1}(x)=\left(\frac{\alpha}{x}-\frac{d}{d x}\right) J_{\alpha}(x)
\end{aligned}
$$

и условия (13). Тогда

$$
\begin{aligned}
& \left\langle k,|m \| \rho| k^{\prime},|m-1|\right\rangle \\
& =\left\{\begin{array}{l}
\left(\frac{m}{k^{\prime}}+\frac{\partial}{\partial k^{\prime}}\right) \frac{\delta\left(k-k^{\prime}\right)}{k}, m>0, \\
\left(\frac{|m|}{k^{\prime}}-\frac{\partial}{\partial k^{\prime}}\right) \frac{\delta\left(k-k^{\prime}\right)}{k}, m \leq 0
\end{array}\right.
\end{aligned}
$$

и

$$
\begin{aligned}
& \left\langle k,|m||\rho| k^{\prime},|m+1|\right\rangle \\
& =\left\{\begin{array}{l}
\left(\frac{m}{k^{\prime}}-\frac{\partial}{\partial k^{\prime}}\right) \frac{\delta\left(k-k^{\prime}\right)}{k}, m \geq 0, \\
\left(\frac{|m|}{k^{\prime}}+\frac{\partial}{\partial k^{\prime}}\right) \frac{\delta\left(k-k^{\prime}\right)}{k}, m<0 .
\end{array}\right.
\end{aligned}
$$

\section{Список литературы}

[1] Woo Jin Kwon, Joon Hyun Kim, Sang Won Seo, Shin Y. // Phys. Rev. Lett. 2016. N 117. P. 245301.

[2] Sasaki K., Suzuki N., Saito H. // Phys. Rev. Lett. 2010. N 104. P. 150404.

[3] Stagg G.W., Allen A.J., Barenghi C.F., Parker N.G. // J. Phys. Conf. Ser. 2015. N 594. P. 012044.

[4] Лойщянский Л.Г. Механика жидкости и газа: учебник для вузов. 7-е изд., испр. М.: Дрофа, 2003. 840 с.

[5] Рабинович М.И. // УФН. 1978. 125. С. 123-168.

[6] Ngoko Djiokap J.M., Hu S.H., Madsen L.B., Manakov N.L., Meremianin A.V., Starace A.F. // Phys. Rev. Lett. 2015. Vol. 115. N 11. P. 113004.

[7] Schmidt L.Ph.H., Goihl C., Metz D., Schmidt-Böcking H, Dörner R., Ovchinnikov S.Yu., Macek J.H., Schultz D.R. // Phys. Rev. Lett. 2014. Vol. 112. P. 083201.

[8] Larionov N.V., Kolobov M.I. // Phys. Rev. A. 2013. V. 88. N 1. P. 013843. DOI: 10.1103/PhysRevA.88.013843; Скалли M.O., Зубайри М.С. Квантовая оптика. Под ред. В.В. Самарцева. М.: Физматлит, 2003. $512 \mathrm{c.}$

[9] Баргатин И.В., Гришанин Б.А., Задков В.Н. // УФН. 2001. 171. С. 625-647; Бауместер Д., Экерт А., Цайлингер А. Физика квантовой информации. Москва: Постмаркет, 2002. $376 \mathrm{c}$.

[10] Ищенко А.А., Асеев С.А., Баграташвили В.Н., Панченко В.Я., Рябов Е.А. // УФН. 2014. 184. С. 681722. DOI: $10.3367 /$ UFNr.0184.201407a.0681; Senaratne R., Rajagopal S.V., Shimasaki T., et al. // Nat. Commun. 2018. V. 9. N 2065. P. 1-7. DOI: 10.1038/s41467-018-04556-3

[11] Овчинников С.Ю., Ларионов Н.В., Смирновский А.А., Шмидт А.A. // Научно-технические ведомости СПбГПУ. Физ.-матем. науки, 2017. Т. 10. № 4. С. 111.

[12] Ngoko Djiokap J.M., Meremianin A.V., Manakov N.L., Hu S.X., Madsen L.B., Starace Anthony F. // Phys. Rev. A. 2016. Vol. 94. N 1. P. 013408.

[13] Ландау Л.Д., Лифиии, E.M. Теоретическая физика в 10 томах. Том 3. Квантовая механика (нерелятивистская теория). Учебное пособие. 6-е изд., испр. М.: Физматлит. 2004. $800 \mathrm{c}$.

[14] Делоне Н.Б., Крайнов В.П. Нелинейная ионизация атомов лазерным излучением, М.: Физматлит. 2001. 311 с.

[15] Parfitt D.G.W., Portnoi M.E. // J. Mathematic. Phys. 2002. Vol. 43. N 10. P. 4681-4691.

[16] Yang X.L., Guo S.H., Chan F.T., Wong K.W., Ching W.Y. // Phys. Rev. A. 1991. Vol. 43. N 3. P. 1186-1196.

[17] Маделунг Э. Математический аппарат физики: справочное руководство. М.: Наука, 1968.

[18] Розанов Н.Н. // Оптика и спектроскопия. 2018. Т. 124. № 1. C. $75-77$. 